\title{
Surface and Underground Water Level Monitoring Using Wireless Sensor Node with Energy Harvesting Support
}

\author{
Zivorad Mihajlovic ${ }^{1}$, Vladimir Milosavljevic ${ }^{1}$, Ana Joza ${ }^{1}$, Vladimir Rajs ${ }^{1}$, \\ Mirjana Damnjanovic ${ }^{1}$, Milos Zivanov ${ }^{1}$ \\ ${ }^{I}$ Faculty of Technical Sciences, University of Novi Sad, \\ Trg Dositeja Obradovica 6, 21000 Novi Sad, Serbia \\ zivorad@uns.ac.rs
}

\begin{abstract}
In this paper development and testing of a wireless sensor node that is powered by solar energy harvesting is described. Implemented wireless sensor node is characterized by low cost and consumption, long mean time between maintenance, simplicity, flexibility, modularity and miniature design in applications for monitoring of environmental parameters. As a replacement for relatively expensive battery supply and in order to minimize maintenance costs, energy harvesting solution that uses a miniature solar panel and supercapacitor is tested. This node is used for measurements of water levels of surface and underground waters for application in agriculture. For this purpose the node is expanded with a capacitive sensor for measurement of water levels, which is particularly discussed in this paper as simple and innovative solution.
\end{abstract}

Index Terms-Capacitive sensor; energy harvesting; wireless sensor networks; environmental monitoring; supercapacitor.

\section{INTRODUCTION}

Research in the field of wireless sensor networks (WSNs) has contributed to many areas such as disaster management, automotive, infrastructure monitoring, security and surveillance, etc. In recent decades, numerous examples of the application of WSNs for environmental monitoring have emerged [1]-[3]. Environmental protection requires enormous financial resources, but potential investors do not find interest to invest since this field is not profitable at the moment. Therefore, the tendency is to invest a minimum of resources in low-cost systems for monitoring of environmental parameters with not optimal, but satisfactory performance. Overall, WSNs for environmental monitoring are considered to be of higher quality if they meet the following requirements: low price, reliable operation in remote field conditions, adaptability to specific application requirements, and a negligible impact on the environment.

The problem of excess waters in the Autonomous Province of Vojvodina, Serbia, is a typical example where employing WSNs could provide information that has an effect on increase in agricultural production. Although during the course of the last two centuries extensive

Manuscript received 19 December, 2015; accepted 16 July, 2016.

This research was funded by the III 43008 project from the Ministry of Science and Education of the Republic of Serbia. measures have been undertaken, there were many climate extremes in the last decades which directly affect on the problem of excess water. Whether due to flooding or due to increased precipitation, a certain part of fertile arable land is unusable. Today, the Vojvodina region has a network of almost $20000 \mathrm{~km}$ of drainage canals [4]. Due to insufficient funding, this drainage network is poorly maintained, which often increases the overall damage caused by natural disasters. Information collected by monitoring of water levels in drainage canals can be used for timely response and prevention in case of future climate extremes or for irrigation management in normal conditions. Generally, continuous measurement of groundwater level on number of points over long periods of time (several years) with usage of hydrodynamic models, some excess water types could be forecasted, and actions could be taken [5].

Successful implementations of WSNs for water level monitoring already exist [5]-[8]. In this paper, a wireless sensor node which is characterized by simplicity, low cost and ease of installation is described. Dedicated hardware design enables ultra-low power consumption in comparison with commercial solutions. In addition, with the selection of special electronic components, the price per node is drastically reduced, as well as dimensions. Since the primary intention is to use a sensor node for measuring of environmental parameters in inaccessible terrains, solar energy harvesting has been added. Using energy harvesting combined with a supercapacitor as a storage element, the battery supply becomes unnecessary, which contributes to maintenance costs reduction. Detailed description of node is given in Section II. In Section III, it is shown how basic node is upgraded with an expansion board for the measurement of small capacitance. The process how to use a simple two-wire cable for measurement of water levels is explained. Section IV shows results of testing a sensor in real application followed by conclusion in Section $\mathrm{V}$.

\section{IMPLEMENTATION OF WIRELESS SENSOR NODE WITH ENERGY HARVESTING SUPPORT}

In practice only wealthy countries spend significant resources for environmental protection. This is the main reason why the minimization of costs is required in the design. One way to achieve this includes design of dedicated 
hardware, resulting in a lower cost of a single sensor node and lower power consumption. The prices of installation and maintenance will be reduced as well. As a consequence the price of whole WSNs will be decreased.

\section{A. Dedicated Hardware Design of the Node}

Based on above defined requirements, a wireless sensor node is designed, and the block diagram of hardware structure is shown in Fig. 1. Control and communication systems are based on commercial components MSP430G2553 microcontroller (MCU) and nRF24L01+ RF transceiver, because they already proved to be an excellent choice for low-power and low-cost WSNs [9]. MSP430G2553 is a mixed signal MCU that is characterized by the following features: very low power consumption, ultra-fast wake-up time from standby mode (less than $1 \mu \mathrm{s}$ ), and a multitude of peripherals. This MCU is a popular choice of embedded system designers, which enables easy prototyping with lots of documentation available.

The low-cost nRF24L01+ from Nordic Semiconductor is a single chip RF transceiver for the global, license-free $2.4 \mathrm{GHz}$ ISM band. It can provide very high speed communications (up to $2 \mathrm{Mbps}$ ) with extremely low power consumption (the RX current is just $13.5 \mathrm{~mA}$ ). Output power, frequency channels, and protocol setup are easily programmable through serial peripheral interface (SPI) of MSP430G2553 MCU. Current consumption for different operating modes is given in Table I.

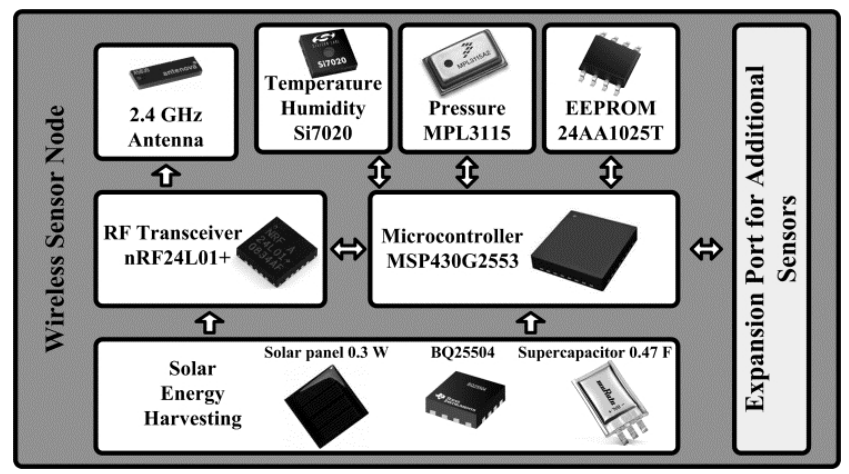

Fig. 1. Hardware subsystems and the most important components incorporated into wireless sensor node.

Since the node is designed for applications in the field of environmental protection, sensors for pressure, temperature and humidity, as well as EEPROM for storing additional data are included in design. By introduction of these sensors writing firmware and node testing in real conditions becomes easier. The sensors are selected in accordance with the requirements of minimum consumption and low cost.

The Si7020-A20 sensor from Silicon Labs consists of precision relative humidity sensor $( \pm 4 \%)$ and high accuracy temperature sensor $\left( \pm 0.4{ }^{\circ} \mathrm{C}\right)$ with $\mathrm{I}^{2} \mathrm{C}$ interface and $3 \times 3 \mathrm{~mm}^{2}$ package size. The MPL3115A2 from Freescale Semiconductor is a MEMS sensor with an $\mathrm{I}^{2} \mathrm{C}$ interface that provides accurate measurements of pressure, altitude and temperature. For additional sensors there is an expansion port available. All MCU interfaces, except SPI, are available on this port. In this paper, the expansion port is used to attach capacitive sensor for water level measurement.

Total cost of developed node is about 20 Euros which is less expensive compared to most commercial solutions [10].
Unfortunately, simpler and less expensive hardware is not suitable for more complex WSNs routing protocols.

TABLE I. CONSUMPTION OF THE MOST IMPORTANT COMPONENTS OF NODE WITH APPROXIMATE PRICES

\begin{tabular}{|c|c|c|c|}
\hline Component & $\begin{array}{l}\text { Operating } \\
\text { Mode }\end{array}$ & Consumption & Price \\
\hline \multirow{2}{*}{ MSP430G2553 } & Active & $230 \mu \mathrm{A}(1 \mathrm{MHz})$ & \multirow{2}{*}{$0.95 €$} \\
\hline & Standby & $0.5 \mu \mathrm{A}$ & \\
\hline \multirow{3}{*}{ NRF24L01+ } & Active & $\begin{array}{l}11.3 \mathrm{~mA} \mathrm{TX} \text { at } 0 \mathrm{dBm} \\
13.5 \mathrm{~mA} \mathrm{RX} \text { at } 2 \mathrm{Mbps}\end{array}$ & \multirow{3}{*}{$1.44 €$} \\
\hline & Standby & $26 \mu \mathrm{A}$ & \\
\hline & Power down & $900 \mathrm{nA}$ & \\
\hline \multirow{2}{*}{ Si7020 } & Active & $150 \mu \mathrm{A}$ & \multirow{2}{*}{$3.12 €$} \\
\hline & Standby & $60 \mathrm{nA}$ & \\
\hline \multirow{2}{*}{ MPL3115 } & Active & $2 \mathrm{~mA}$ & \multirow{2}{*}{$1.82 €$} \\
\hline & Standby & $2 \mu \mathrm{A}$ & \\
\hline \multirow{2}{*}{ 24AA1025T } & Active & $450 \mu \mathrm{A}$ & \multirow{2}{*}{$2.57 €$} \\
\hline & Standby & $5 \mu \mathrm{A}$ & \\
\hline BQ25504 & Quiescent & $330 \mathrm{nA}$ & $2.45 €$ \\
\hline
\end{tabular}

With a consumption of about $4 \mu \mathrm{A}$ (without EEPROM) in standby operating mode and average consumption of about few milliamps in active mode (without communication task), implemented node is very good low-power solution and can be considered as better, compared to some commercial solutions [10]-[12]. However, since our main objective is to measure different environmental parameters, it is necessary to include consumption of sensors attached to the node. Although the tendency is to reduce the consumption of the sensor as much as possible, in practice this is often impossible. Also, when measuring certain parameters, it is necessary to supply sensors for a period of time (e.g. heating of gas sensors). Battery supply in such cases will be quickly depleted. Energy harvesting is an efficient replacement for batteries in the field of environmental monitoring.

\section{B. Implementation of Solar Energy Harvesting}

In addition to the low price and consumption, an important requirement for node design is to minimize maintenance costs after installation of WSNs. Commercial nodes generally use some form of battery power, which, depending on the node type, can last for a very long time, even up to several years. The batteries as limited energy source must be optimally used for both processing and communication tasks. Since communication task tends to dominate over the processing task in order to make optimal use of energy, the amount of communication should be reduced as much as possible. In practical applications, the wireless sensor nodes are usually deployed in hostile or unreachable terrains where they cannot be easily retrieved for the purpose of replacing or recharging the batteries, therefore the lifetime of the network is usually limited. When the battery is finally depleted, it is necessary to invest additional resources in their replacement and expensive field work in harsh conditions for recovery of relatively large number of nodes. Furthermore, the batteries themselves increase the cost, especially if the specific battery types with high capacity and low self-discharge current are used.

Given the above, it can be concluded that in order to reduce cost of WSNs, it is necessary to find a way to minimize maintenance costs. The easiest way to reduce costs is to increase the time between two battery 
replacements. This can be achieved in two ways. Firstly, the capacity of energy source should be increased, but dimensions of the node must remain the same. In other words, the energy density of the source must be increased, but unfortunately the technology of battery production is limited at the moment. Secondly, the power consumption should be minimized, either by selecting more efficient components or by firmware optimization. In many cases, hardware implementation is already optimized for ultra-low power application, and by firmware optimization only slight improvements can be achieved.

In this paper, the third option that includes energy harvesting is considered, allowing a node to generate its own power from the ambient. Energy harvesting involves techniques that capture, harvest or scavenge unused ambient energy (such as kinetic, thermal or electromagnetic) and convert it into usable electrical energy that is stored and later used for node operation. Energy harvesting subsystem consists of energy source, energy harvester, power management circuit and energy storage element (Fig. 1). More information about energy harvesting technologies, models and possible applications can be found in [12]-[15].

In this paper, harvesting subsystem with the miniature solar panel (photovoltaic cell) as energy source was used. Energy harvesting with photovoltaic cells is popular and well-studied [16]-[19]. Photovoltaic cells are the most popular choice because of their availability and relatively high performance in comparison to the other energy harvesting techniques [17]. However, solar power technology is still expensive, so it is important to maximize the power output. In order to improve the efficiency of photovoltaic cell, methods such as maximum power point tracking (MPPT) are used. There are many types of MPPT techniques. These techniques differ in many aspects, including complexity, convergence speed, hardware implementation, sensors required, cost, range of effectiveness and need for parameterization [20]. MPPT circuit operates on the basis of the maximum power transfer theorem to extract as much power as possible by impedance matching, in order to compensate for the varying characteristic resistance of solar panels, thus providing higher power outputs.

In our implementation, energy harvesting circuit based on an integrated chip BQ25504 from Texas Instruments is used. The BQ25504 is an ultra-low power charging controller intended for interfacing DC sources such as solar cells, thermal generators and high-impedance batteries. Controller uses the open-circuit voltage (OCV) technique to regulate the solar cell to its maximum power point (MPP) [20]. The MPPT circuit obtains a new reference voltage every $16 \mathrm{~s}$ (typical) by periodically disabling the charger for $256 \mathrm{~ms}$ (typical) and sampling a fraction of the harvester's opencircuit voltage. For solar harvesters, the MPP is typically $70 \%-80 \%$ of open-circuit voltage. For the solar panel the output power in function of voltage is not linear function [18]. The exact ratio for MPPT can be optimized to meet the needs of the input source being used by connecting external resistors divider. BQ25504 controller is an all in one solution for energy harvesting, but it requires an understanding of the settings for a successful implementation. Among other parameters it is possible to adjust the undervoltage threshold (VBAT_UV) to prevent storage component to be deeply discharged or damaged (Fig. 2). Also, the overvoltage protection (VBUT_OV) is configurable. As information that the controller is ready and that the voltage has reached the operational value a digital output signal (VBAT_OK) is used. This signal can awake MCU from sleep operating mode by using interrupt service routine. Both signal level and hysteresis (HYST) are easily configurable via external resistors. The load is not directly connected to the storage element, but internally via a MOSFET. The controller on the basis of the external resistors values and internal references regulates the flow of power to and from the storage element. The whole process is shown in Fig. 2. When the input voltage of controller exceeds the minimum input voltage with sufficient power, the cold-start subsystem turns on. The cold-start subsystem is essentially an unregulated boost converter. When the storage capacitor voltage reaches $1.8 \mathrm{~V}$, the main boost regulator starts up, and charging the storage element begins when the output voltage of the controller reaches VBAT_UV_HYST $(2.10 \mathrm{~V}+80 \mathrm{mV})$. The load is being supplied when output voltage reaches VBAT_OK_HYST $(2.45 \mathrm{~V})$. Controller turns off when it reaches VBAT_OV $(3.53 \mathrm{~V})$, when the overvoltage protection is activated. In the opposite direction, when the output voltage drops below overvoltage threshold VBAT_OV_HYST (3.53 V-35 mV) charging resumes. When output voltage drops below VBAT_OK $(2.20 \mathrm{~V})$, the load is separated from the controller, but the charging continues until the output voltage falls below VBAT_UV $(2.10 \mathrm{~V})$. The adjusted voltage range $(2.20 \mathrm{~V}$ to $3.53 \overline{\mathrm{V}})$ corresponds to the working voltage range of MSP430G2553 (2.00 V to $3.60 \mathrm{~V})$. In this way the energy in the storage element is maximally utilized.

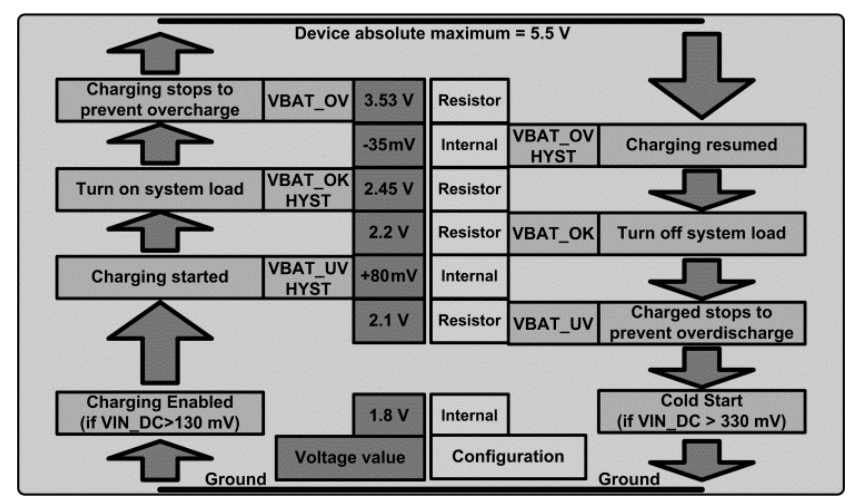

Fig. 2. Relative positions of the threshold voltages that affect charging/discharging of the storage element.

\section{Supercapacitor as Storage Element}

The node described in this paper is designed for use in the field conditions for monitoring of environmental parameters. Most of the environmental parameters are changing slowly, which means that frequent measurement is not necessary. When measurement of surface and underground water levels is considered, situation is even more favourable. Under normal conditions when there are no serious changes in water levels, sending data several times per day is sufficient. In the case of sudden changes in water levels, the node sends emergency information. Therefore, a large capacity power source is not necessary.

For requirements of low power consumption and low 
frequency data transmissions, supercapacitor is chosen as energy storage. Supercapacitor technology is constantly evolving, so their application increases as well. More details about the analysis and application of supercapacitor in WSNs can be found in [21]-[22]. In our hardware implementation Murata supercapacitor with capacitance of $0.47 \mathrm{~F}$ and nominal voltage of $4.20 \mathrm{~V}$ is used (Fig. 1). Miniature dimensions, especially small thickness are suitable for the design of multi-layered modular structures.

The maximum consumption of node is practically equal to the consumption of the communication module (11.30 mA for transmission). The node consumption in standby operating mode is less than $4 \mu \mathrm{A}$. For supercapacitor that discharges from $3.53 \mathrm{~V}$ to $2.20 \mathrm{~V}$ (Fig. 2), testing has shown that the node is operational for several days without charging in a case when the communication task is executed once per hour. The changes of voltage in case when communications occur once per second, once per ten seconds and once per ten minutes are shown in Fig. 3. In this experiment the supercapacitor is charged to the maximum value $(3.53 \mathrm{~V})$, and then the solar panel is disconnected. The MCU is awakened in exactly specified intervals when the voltage on the supercapacitor is measured and then sends the measured value via radio communication. Upon the completion of transmission, the MCU returns to sleep operating mode.

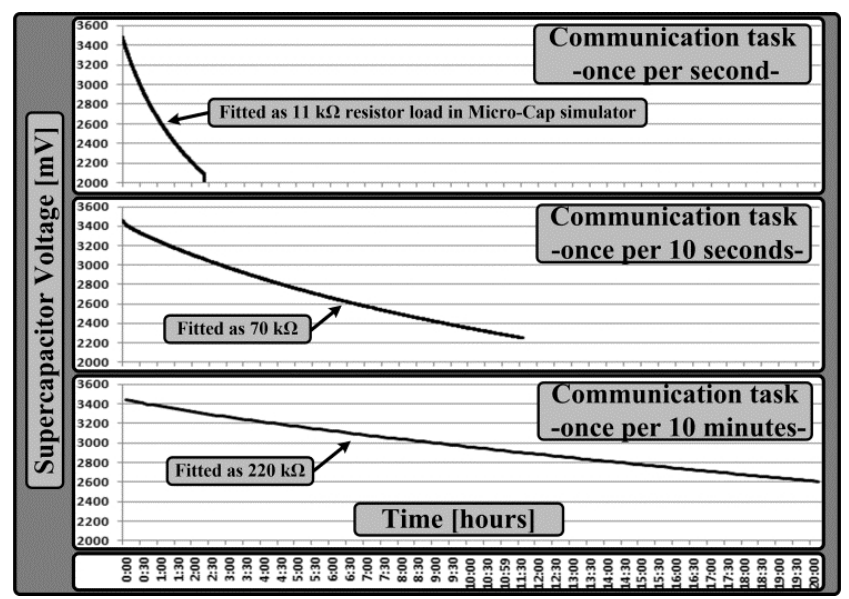

Fig. 3. Measured voltage on supercapacitor for different frequencies of communication task. Equivalent resistances of $11 \mathrm{k} \Omega, 70 \mathrm{k} \Omega$ and $220 \mathrm{k} \Omega$ are calculated with Micro-Cap simulator.

It may be noted that in the case of sending data once per 10 seconds, the energy from the supercapacitor is sufficient to supply the sensor node almost 12 hours. For less frequent transmission, energy from the supercapacitor is sufficient for the operation during a few days or even more. Of course, this mode will not occur in practice because the node will be recharged at the first light in the morning.

Using Micro-Cap circuit simulator, supercapacitor voltage curves fitted to experimental data are obtained. Average power consumption is modelled with resistor whose value depends on frequency of communication task. Calculated values are $11 \mathrm{k} \Omega, 70 \mathrm{k} \Omega$ and $220 \mathrm{k} \Omega$ for frequencies of communication tasks shown in Fig. 3. This simplifies estimation of supercapacitor discharge time. Also, in the reverse direction equivalent resistance can be used to calculate the optimum frequency of the communication task.

Recharging time of supercapacitor for several different light intensities is shown in Fig. 4. The worst case of supercapacitor charging is during period before sunset and when it is cloudy. Even then, less than 5 minutes is enough to supercapacitor to be charged at full capacity. At sunny day, around $20 \mathrm{~s}$ for charging is enough.

It can be concluded that the supercapacitor will be certainly recharged during the day. In practice, the capacitor is charged to the maximum value for most of the day, even when the node is continuously sending data and reading sensors. The voltage of the supercapacitor within a period of two days with communication task executed once per minute is shown in Fig. 5. It should be noted that the experiment was carried out in mid-December when the day is the shortest (in the presence of fog also), so this is the worst possible scenario that may occur during the year.

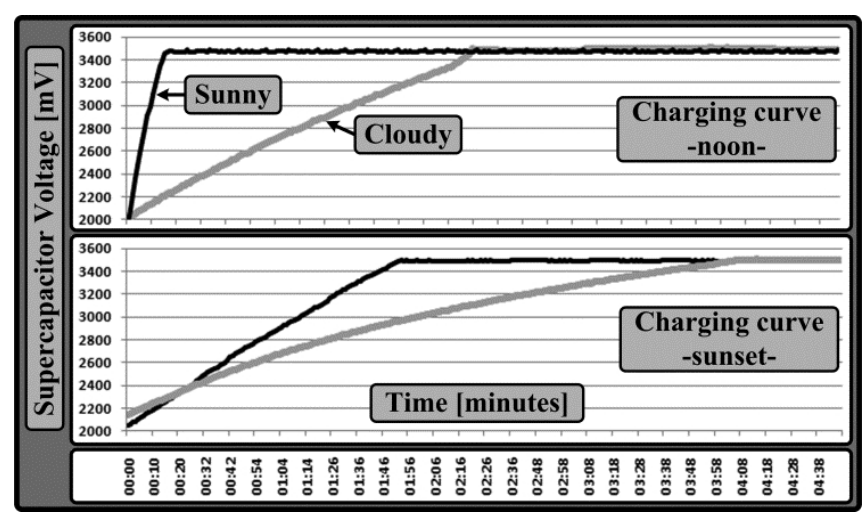

Fig. 4. The charging times of supercapacitor for different light intensities.

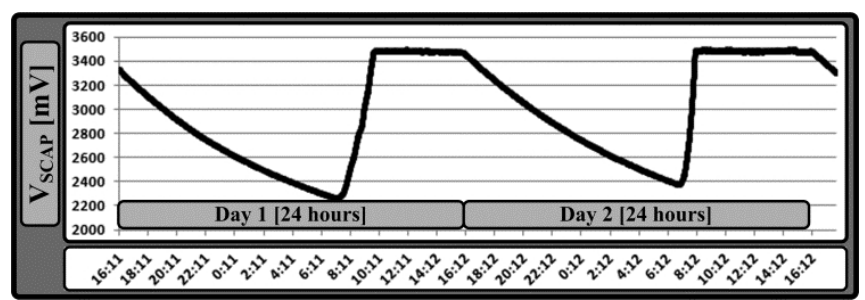

Fig. 5. The voltage of the supercapacitor within a period of two days with communication task executed once per minute.

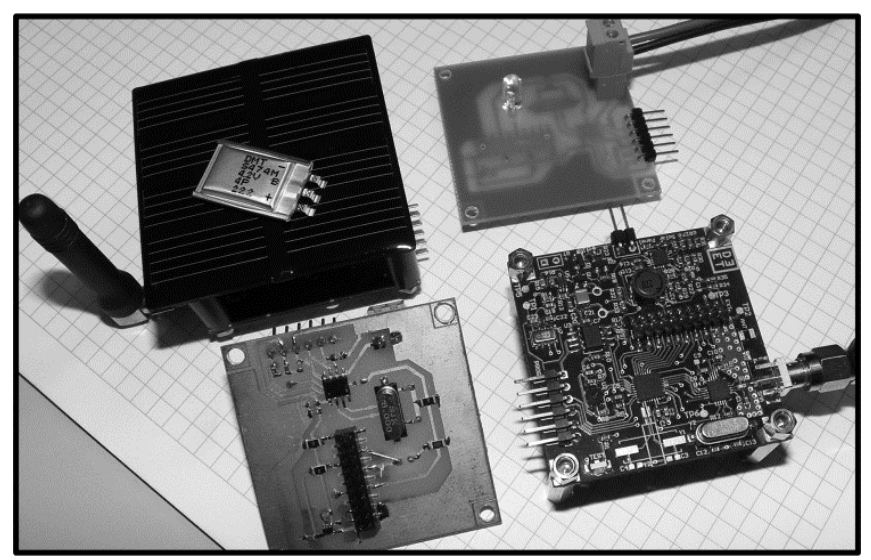

Fig. 6. Photo of wireless sensor node with energy harvesting extended with module for measurement of very small capacitance.

The conclusion is that the designed node can be used without restrictions if there is daylight, but during the night it is necessary to save energy to last until a new charging cycle. Also, during the daylight it is possible to use sensors that require a greater amount of energy (e.g. gas sensors that require heating). The photo of realized node, expansion board, solar panel and supercapacitor is shown in Fig. 6. 


\section{CAPACITIVE SENSOR FOR WATER LEVEL MEASUREMENT}

For water level measurement simple expansion board based on PIC12F1822 MCU from Microchip is designed (Fig. 6). In order to reduce the consumption, a load switch integrated circuit TPS22944 from Texas Instruments is used. The main MCU uses switch to control power supply of the extension board. When measurement of the water level is not performed, the consumption of the hardware section that executes this task is practically reduced to zero. When using limited resources of alternative energy, this represents a significant advantage in node's performance. This is a recommended method to extend functionality of the node.

The measurement setup where expansion board is added to wireless sensor node is shown in Fig. 7. PIC12F1822 has integrated capacitive sensing (CPS) module, which is suitable for measuring small capacitance values. Two-wire cable, which represents a capacitive sensor, is added to the expansion board. Capacitance sensing method is chosen due to its reliable operation, low cost and because it has no moving parts. Two-wire cable acts as a water level probe that is immersed inside water and it can be made from any cable type that is isolated at bottom side. This water level probe is connected to MCU's CPS module. No signal conditioning electronics is needed between sensing probe and MCU which makes hardware design extremely simple.

Capacitance of isolated cable partially immersed in water is read by MCU's CPS oscillator module. Cable acts as the capacitor $\left(C_{C A B L E}\right)$, which capacitance changes linearly with length of the cable section immersed in water. Dielectric constant around cable changes $\left(\varepsilon_{r}\right)$, which results in the equivalent cable capacitance change. The time dependence of the capacitor voltage $\left(V_{C A B L E}\right)$ can be described with

$$
V_{C A B L E}\left(t_{1}\right)=V_{C A B L E}\left(t_{0}\right)+\frac{1}{C_{C A B L E}} \times \int_{t_{0}}^{t_{1}} i(t) \times d t \text {. }
$$

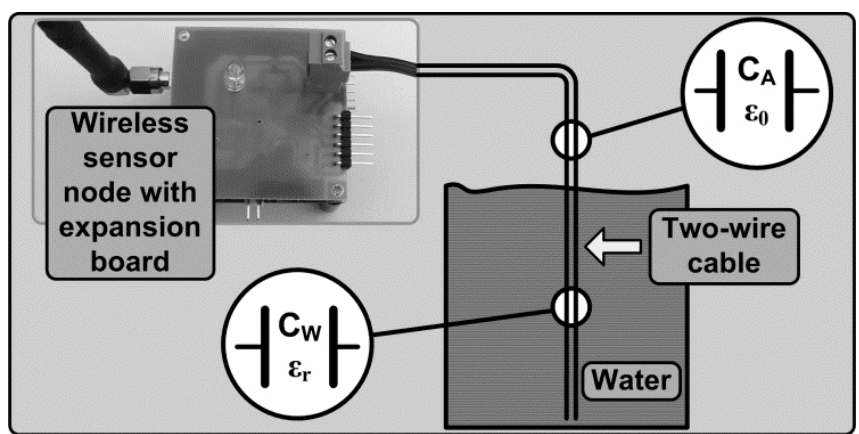

Fig. 7. Wireless sensor node with expansion board that measures the capacitance of two-wire cable immersed in water.

By using constant current (1) is simplified to

$$
V_{C A B L E}\left(t_{1}\right)=V_{C A B L E}\left(t_{0}\right)+\frac{I_{C P S}}{C_{C A B L E}} \times\left(t_{1}-t_{0}\right) .
$$

If we take into account the fact the charging and discharging currents $\left(I_{C P S}\right)$ are the same, by measuring the frequency of oscillation of the CPS oscillator, we can express rise $\left(t_{R I S E}\right)$ and fall time $\left(t_{F A L L}\right)$ as

$$
t_{F A L L}=t_{R I S E}=\frac{1}{2 \times f_{C P S}} .
$$

Cable capacitance per unit length is specified by manufacturer, in the air $\left(C_{A}^{\prime}\right)$ is $55.13 \mathrm{pF} / \mathrm{m}$, and in the water $\left(C^{\prime}{ }_{W}\right)$ is $296.33 \mathrm{pF} / \mathrm{m}$.

The total capacitance to be measured is:

$$
\begin{gathered}
C_{E K V}=C_{0}+L_{A} \times C_{A}^{\prime}+L_{W} \times C_{W}^{\prime}, \\
C_{E K V}=C_{0}+\left(L_{C}-L_{W}\right) \times C_{A}^{\prime}+L_{W} \times C_{W}^{\prime},
\end{gathered}
$$

where $C_{0}$ denotes parasitic capacitance of the PCB tracks and the cable connector, $L_{A}$ is the length of the cable in the air, $L_{W}$ is the length of the cable immersed in the water and $L_{C}$ is the total length of the cable. The firmware measures the operating frequency of the CPS oscillator. The total measured capacitance $\left(C_{E K V}\right)$ can be determined using

$$
C_{E K V}=\frac{I_{C P S} \times\left(t_{1}-t_{0}\right)}{V_{C}\left(t_{1}\right)-V_{C}\left(t_{0}\right)},
$$

where $t_{1}$ represents the moment of reaching a positive reference voltage of CPS oscillator, while $t_{0}$ is the moment of reaching negative reference voltage of CPS oscillator. Thus (6) comes down to

$$
C_{E K V}=\frac{I_{C P S} \times t_{R I S E}}{V_{+}-V_{-}}=\frac{I_{C P S}}{\left(V_{+}-V_{-}\right) \times 2 f_{C P S}} .
$$

In this paper the following configuration of CPS oscillator is used: $V_{+}=2048 \mathrm{mV}$ which is the internal reference voltage and $V_{-}=10 / 32 \cdot 2048 \mathrm{mV}$.

The negative reference is obtained by using an internal 5bit DAC converter which also uses the internal reference voltage of $2048 \mathrm{mV}$. The current source of CPS oscillator is adjustable by software and is set to $9 \mu \mathrm{A}$.

Substituting these values in (7), the expression for the capacitance of the sensor cable is calculated with

$$
C_{E K V}=\frac{3.196 \times 10^{-6}}{f_{C P S}} \text {. }
$$

Using (5), the water level can be calculated, i.e. the length of cable immersed in water

$$
L_{W}=\frac{C_{E K V}-C_{0}-L_{C} C_{A}^{\prime}}{C_{W}^{\prime}-C_{A}^{\prime}} .
$$

The reference capacitor of $470 \mathrm{pF}$ capacitance and 5\% tolerance was added on the expansion board for testing purposes. In this case due to the short conductive track lines $C_{0}$ can be neglected. By measuring the frequency of the CPS oscillator for this channel, the value of $6885 \mathrm{~Hz}$ is obtained and using (8) the measured capacitance value of $464.20 \mathrm{pF}$ is calculated. This value represents a deviation of $1.27 \%$ that is within the tolerance value of testing capacitor used.

\section{RESULTS AND ANALYSIS}

The device was tested in a calm section of the Danube 
River. As a water level probe a $200 \mathrm{~cm}$ long cable is used. Sensing cable is marked every $10 \mathrm{~cm}$ and immersed into the water. The variation of water level was accomplished by changing the depth of cable immersion in the steps of $10 \mathrm{~cm}$. For each marked point minimum 10 measurement results are recorded. Substituting the known parameters of the sensor cable in (9), a graph in Fig. 8 is produced. It shows the deviation of the measured from expected capacitance value. During measurements the adjustment of the level to which the cable is immersed in water was done manually, so there is an impact of human factor on the measurement errors which is shown as error in centimetres of full scale in Fig. 8. This graph shows that the largest error occurs at higher values of the immersion depth of the cable. This can be explained by the influence of human error during the device calibration in inconvenient field conditions and difficult access to water in the measurement range. The main disadvantage of this solution is the recovery time after removing a sensor cable out of the water. Due to the remaining water drops and moisture on the cable surface after extraction a false reading may occur. The impulse response of the system is determined by immersing the entire length of the sensor cable into the water and subsequent rapid withdrawal of the entire cable. This impulse response is shown in Fig. 9. Considering that this response is noticeable only when the cable is removed from water and only for drastic water level changes, we come to the following conclusions. System response during immersion in water is available immediately. When removing the cable from the water the response is exponential. The measured signal reaches $95 \%$ of the asymptotic value in the first $5 \mathrm{~s}$ of measurement. This system is efficient for slowly varying surface water levels.

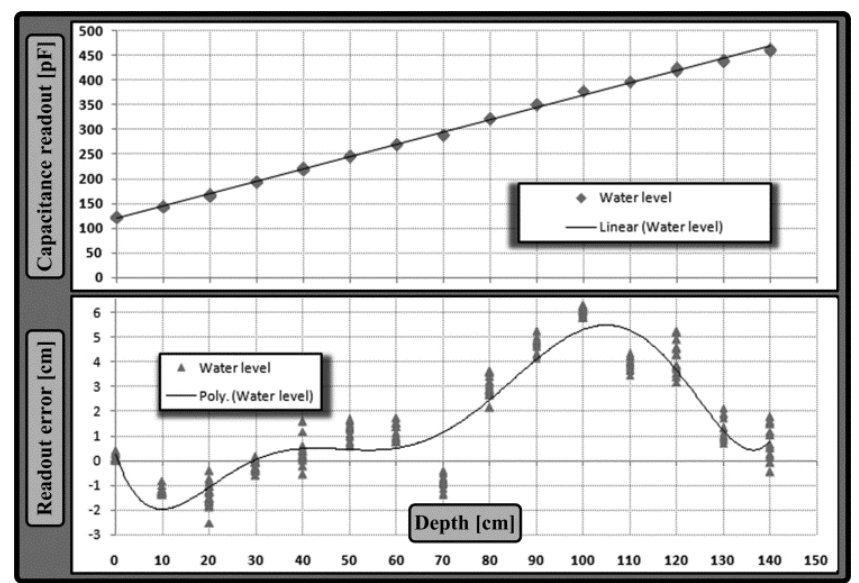

Fig. 8. Measured and expected values versus immersion depth and absolute measurement error during calibration.

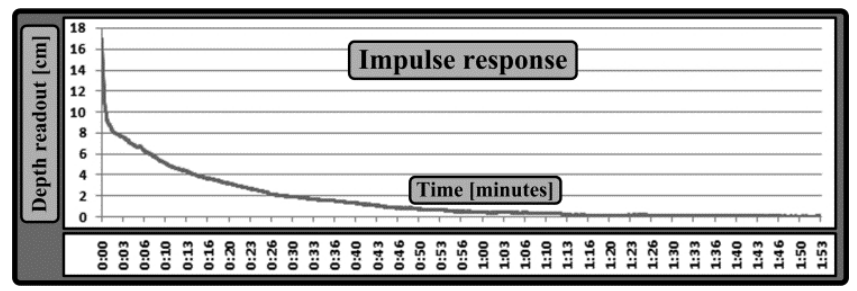

Fig. 9. Water sensor response for sudden water level change.

The purpose of this wireless sensor node is monitoring of water level in slowly varying systems, and the slow response during the decline of the water level has no greater influence on the measurement results. When water level changes fast, this method is not recommended.

\section{CONCLUSIONS}

Implemented low-power and low-cost wireless sensor node uses energy harvesting from miniature solar panel and supercapacitor as storage element. Experiments have shown that the energy stored in the supercapacitor is sufficient for the autonomous operation of the sensor node if smart management of communication tasks is used. Also, experiments have shown that the supercapacitor will be certainly recharged during the day. The absence of batteries drastically reduces the cost of the node and its maintenance, which enables application in difficult and inaccessible terrain. One such application is the measurement of water levels of surface and ground waters that threaten agricultural production. For this application, the functionality of the sensor node with specially designed capacitive sensor has been upgraded. The sensor is effective when the water level rises, which is very important in case of an emergency. When the water level decreases, the response is slightly slower. This is not critical, since the decline is usually slow.

\section{REFERENCES}

[1] C. See, K. Horoshenkov, R. Abd-Alhameed, Y. Hu, "A low power wireless sensor network for gully pot monitoring in urban catchments", IEEE Sensors J., vol. 12, pp. 1545-1553, 2011.

[2] R. L. Pereira, J. Trindade, F. Goncalves, L. Suresh, D. Barbosa, T. Vazao, "A wireless sensor network for monitoring volcano-seismic signals", Natural Hazards and Earth System Sciences, vol. 14, no. 12 pp. 3123-3142, 2014. [Online]. Available: http://dx.doi.org/10.5194/ nhess-14-3123-2014

[3] M. Pies, R. Hajovsky, S. Ozana, “Autonomous monitoring system for measurement of parameters of heat collection technology at therma active mining dumps", Elektronika Ir Elektrotechnika, vol. 19, no. 10 pp. 62-65, 2013. [Online]. Available: http://dx.doi.org/10.5755/ j01.eee.19.10.5898

[4] A. Belic, R. Savic, S. Belic, "Living with water in Vojvodina", IAHS Publication, vol. 286, pp. 178-183, 2004

[5] M. Brkic, D. Obradovic, V. Dogan, B. Karoly, M. Zivanov, "Acoustic measurement and monitoring system for level of groundwater", Sensor Letters, vol. 11, no. 9, pp. 1-5, 2013. [Online]. Available: http://dx.doi.org/10.1166/s1.2013.2948

[6] F. Cao, F. Jiangy, Z. Liu, "Application of ISFET microsensors with mobile network to build IOT for water environment monitoring", Int. Conf. on Intelligent Environments (IE), 2014, pp. 207-210. [Online]. Available: http://dx.doi.org/10.1109/ie.2014.38

[7] K. Kuang, S. Quek, M. Maalej, "Remote flood monitoring system based on plastic optical fibres and wireless motes", Sensors and Actuators A, vol. 147, no. 2, pp. 449-455, 2008. [Online]. Available: http://dx.doi.org/10.1016/j.sna.2008.05.030

[8] P. Jiang, H. Xia, Z. He, Z. Wang, "Design of a water environment monitoring system based on wireless sensor networks", Sensors, vol. 9, no. 8, pp. 6411-6434, 2009. [Online]. Available: http://dx.doi.org/10.3390/s90806411

[9] S. S. Sonavane, V. Kumar, B. P. Patil, "MSP430 and nRF24L01 based wireless sensor network design with adaptive power control", ICGST-CNIR Journal, vol. 8, pp. 11-15, 2009.

[10] M. Johnshon, M. Healy, P. V. D. Ven, M. J. Hayes, J. Nelson, T. Newe, E. Lewis, "A comparative review of wireless sensor network mote technologies", in Proc. IEEE Sensors Conf., 2009, pp. 1439-1442.

[11] C. Park, J. Liu, P. Chou, "Eco: an ultra-compacy low-power wireless sensor node for real-time motion monitoring", in Proc. 4th Intl. Conf. on Information Processing in Sensor Networks, 2005. [Online] Available: http://dx.doi.org/10.1109/ipsn.2005.1440956

[12] J. M. Gilbert, F. Balouchi, "Comparison of energy harvesting systems for wireless sensor networks", Int. Journal of Automation and Computing, vol. 5, no. 4, pp. 334-337, 2008. [Online]. Available: http://dx.doi.org/10.1007/s1 1633-008-0334-2

[13] S. Sudevalayam, P. Kulkarni, "Energy harvesting sensor nodes: survey and implications", IEEE Communications Surveys \& 
Tutorials, vol. 13, no. 3, pp. 443-461, 2011. [Online]. Available: http://dx.doi.org/10.1109/SURV.2011.060710.00094

[14] R. Shigeta, T. Sasaki, D. M. Quan, Y. Kawahara, R. J. Vyas, M. M Tentzeris, T. Asami, "Ambient RF energy harvesting sensor device with capacitor-leakage-aware duty cycle control", IEEE Sensors Journal, vol. 13, no. 8, pp. 2973-2983, 2003. [Online]. Available: http://dx.doi.org/10.1109/JSEN.2013.2264931

[15] W. K. G. Seah, Z. A. Eu, H. P. Tan, "Wireless sensor networks powered by ambient energy harvesting (WSN-HEAP) - survey and challenges", Wireless VITAE, pp. 1-5, 2009. [Online]. Available: http://dx.doi.org/10.1109/wirelessvitae.2009.5172411

[16] C. Alippi, C. Galperti, "An adaptive system for optimal solar energy harvesting in wireless sensor network nodes", IEEE Trans. Circuits and Systems I: Regular Papers, vol. 55, no. 6, pp. 1742-1750, 2008. [Online]. Available: http://dx.doi.org/10.1109/TCSI.2008.922023

[17] Z. G. Wan, Y. K. Tan, C. Yuen, "Review on energy harvesting and energy management for sustainable wireless sensor networks", IEEE 13th Int. Conf. on Comm. Technology, 2011, pp. 362-367. [Online]. Available: http://dx.doi.org/10.1109/icct.2011.6157897
[18] D. Brunelli, C. Moser, L. Thiele, L. Benini, "Design of a solar harvesting circuit for batteryless embedded systems", IEEE Trans. Circuits Syst. II, vol. 56, no. 11, pp. 2519-2528, 2009. [Online]. Available: http://dx.doi.org/10.1109/TCSI.2009.2015690

[19] A. Nasiri, S. Zabalawi, G. Mandic, "Indoor power harvesting using photovoltaic cells for low-power applications", IEEE Trans. on Industrial Electronics, vol. 56, no. 11, pp. 4502-4509, 2009. [Online]. Available: http://dx.doi.org/10.1109/TIE.2009.2020703

[20] B. Subudhi, R. Pradhan, "A comparative study on maximum powe point tracking techniques for photovoltaic power systems", IEEE Trans. Sustainable Energy, vol. 4, no. 1, pp. 89-98, 2013. [Online]. Available: http://dx.doi.org/10.1109/TSTE.2012.2202294

[21] C. Park, P. H. Chou, "AmbiMax: autonomous energy harvesting platform for multi-supply wireless sensor nodes", Sensor and Ad Hoc Comm. and Networks (SECON), 2006, pp. 168-177.

[22] S. Kim, P. H. Chou, "Size and topology optimization for supercapacitor-based sub-watt energy harvesters", IEEE Trans. Power Electronics, vol. 28, pp. 2068-2080, 2012. [Online]. Available: http://dx.doi.org/10.1109/TPEL.2012.2203147 